\title{
Prognostic Value of Optic Nerve Sheath Diameter in Patients after Extracorporeal Cardiopulmonary Resuscitation
}

\author{
Youngjin $\mathrm{Kim}^{1}$, Jeong-Am Ryu ${ }^{1,2}$, Yong Oh $\mathrm{Kim}^{2}$, Yang Hyun $\mathrm{Cho}^{3}$, Jeong Hoon Yang ${ }^{2,4}$, Sangkil Lee ${ }^{5}$, \\ Ji Sun Baek ${ }^{6}$ \\ ${ }^{1}$ Department of Neurosurgery, Samsung Medical Center, Sungkyunkwan University School of Medicine, Seoul, Korea \\ ${ }^{2}$ Department of Critical Care Medicine, Samsung Medical Center, Sungkyunkwan University School of Medicine, Seoul, Korea \\ ${ }^{3}$ Department of Thoracic and Cardiovascular Surgery, Samsung Medical Center, Sungkyunkwan University School of Medicine, Seoul, Korea \\ ${ }^{4}$ Division of Cardiology, Department of Medicine, Samsung Medical Center, Sungkyunkwan University School of Medicine, Seoul, Korea \\ ${ }^{5}$ Department of Neurology, ChungBuk National University Hospital, Cheongju, Korea \\ ${ }^{6}$ Department of Ophthalmology, Konyang University, Kim’s Eye Hospital, Myung-Gok Eye Research Institute, Seoul, Korea
}

Received: March 19, 2019

Accepted: March 26, 2019

Corresponding Author:

Ji Sun Baek, M.D.

Department of Ophthalmology,

Konyang University, Kim's Eye

Hospital, Myung-Gok Eye

Research Institute, 136 Yeongsin-

ro, Yeongdeungpo-gu, Seoul

07301, Korea

Tel: +82-2-2639-7812

Fax: $+82-2-2633-3976$

E-mail: yangchi100@kimeye.com

\section{Objective}

To determine whether optic nerve sheath diameter (ONSD) could be used to predict neurological outcomes in patients after extracorporeal cardiopulmonary resuscitation (ECPR).

\section{Methods}

This was a retrospective and observational study of adult unconscious patients who were evaluated by brain computed tomography (CT) scan within 48 hours after ECPR between May 2010 and December 2016. ONSDs were estimated on initial brain CT. The primary outcome was neurological status upon hospital discharge assessed with Cerebral Performance Categories (CPC) scale. CPC scores of 1 and 2 indicated good neurological outcome while CPC scores of 3, 4, and 5 indicated poor neurological outcome.

\section{Results}

Among 52 unconscious patients, survival to discharge was identified in 19 (36.5\%) patients. Of these 19 survivors, 13 (25.0\%) had good neurological outcome (CPC score of 1 or 2). ONSDs and their modification in the poor neurological outcome group were significantly greater than those in the good neurological outcome group (all $p \leq 0.003$ ). However, eyeball transverse diameter was not significantly different between the two groups $(\mathrm{p}=0.770)$. Analysis of the receiver operating characteristic curves for predicting poor neurological outcome showed that ONSDs had considerable predictive value (C-statistics: 0.735 to 0.812 ). In addition, ONSDs and their modification had similar performances for predicting poor neurological outcome.

Conclusion

ONSDs measured with CT may be used to predict neurological outcomes of ECPR survivors.

Keywords: Brain computed tomography; Optic nerve sheath diameter; ardiopulmonary resuscitation; Extracorporeal membrane oxygenation 


\section{INTRODUCTION}

Recently, extracorporeal cardiopulmonary resuscitation (ECPR) has been increasingly utilized to supply oxygenated blood and hemodynamic support in the absence of spontaneous cardiac circulation ${ }^{15}$. Neurological outcome is an important issue in patients who have survived cardiac arrest. In these survivors, several predictors of neurological outcomes such as physical examination, several biomarkers, and electrophysiologic studies have been reported ${ }^{19,25)}$. Similar to studies on conventional cardiopulmonary resuscitation (CPR), there have been several studies on neurological prognosis of patients receiving $\mathrm{ECPR}^{11,12,18)}$. However, there are limited data on prognostic imaging markers of neurological outcomes after ECPR ${ }^{15)}$.

After cardiac arrest, primary and secondary brain injuries are associated with intracranial hypertension ${ }^{21}$. Therefore, early monitoring of intracranial hypertension is important as it may allow prediction of neurological outcomes in these patients. Optic nerve sheath diameter (ONSD) has been proposed as an alternative measure for the detection of intracranial hypertension ${ }^{6,22)}$. In addition, ONSD on initial brain computed tomography (CT) may be correlated with neurologic outcomes in conventional CPR setting ${ }^{6,9}$. However, whether ONSD may facilitate systemic evaluation of neurological outcomes of survivors after ECPR has not been reported yet. Therefore, the objective of this study was to investigate if ONSD with some modifications could be used to predict neurological outcomes of patients who underwent ECPR.

\section{METHODS}

\section{Study population}

This was a retrospective and observational study of adult patients who underwent ECPR during hospitalization at Samsung Medical Center between May 2010 and December 2016. This study was approved by the Institutional Review Board of Samsung Medical Center (SMC 2017-11-088-002). The requirement for informed consent was waived due to its retrospective nature. Clinical and laboratory data were collected by a trained study coordinator using a standardized case report form. Patients who underwent ECPR during the study period were included. Those who were unconscious (a score of $<9$ on Glasgow Coma Scale) ${ }^{16)}$ on admission to the hospital after cardiac arrest and those who were given brain CT scan within 48 hours after ECPR were selected. Of these patients, we excluded patients who were under 18 years of age, those who had malignancy whose expected life span was less than one year, those who had insufficient medical records, and those who had a history of head trauma, neurosurgery, or chronic neurological abnormality on ICU admission.

\section{Definitions and outcomes}

In this study, ECPR was defined as both successful veno-arterial extracorporeal membrane oxygenation (ECMO) implantation and pump-on with cardiac massage during index procedure in patients with cardiac arrest. When a return of spontaneous circulation (ROSC) occurred during ECMO cannulation, practitioners typically did not remove the cannula or stop the ECMO pump-on process ${ }^{2,13}$. ECMO pump-on was defined by stopping chest compressions following successful ECMO implantation and activation. ECMO flow was then gradually increased until the patient was hemodynamically stable. Resuscitation was performed in the same way as described in our previous study ${ }^{16,17)}$. Arrest to ECMO pump-on time was defined as the time from cardiac arrest to the time at which the ECMO pump was turned on. Targeted temperature management (TTM) was performed with surface cooling devices. A commercial temperature regulation system consisting of a hydrogel pad (Arctic Sun ; Medivance Corp, Louisville, CO, USA) or a cooling blanket was used. Surface cooling and the degree of targeted temperature were determined by each intensivist in the ICU according to the therapeutic hypothermia protocol ${ }^{7}$. The primary outcome was neurological status upon hospital discharge assessed with Glasgow-Pittsburgh Cerebral Performance Categories (CPC) scale (score of 1 to 5$)^{4}$. CPC scores of 1 and 2 indicated good neurological outcome while CPC scores of 3, 4, and 5 indicated poor neurological outcome. Medical records were thoroughly reviewed. Patients were graded on the CPC scale by two independent neurologists.

All recorded brain CT scans were taken within 48 hours after ROSC in this study. After successful ECPR, for patients who had a rapid recovery of mentality and neurological deficits, brain CT scan was not performed. Otherwise, brain CT scan was performed to determine whether control was needed for increased intracranial pressure (ICP). Brain CT scan was also used to exclude intracranial hemorrhage before therapeutic hypothermia by an intensivist. For all CT studies, 64-channel scanners (Light Speed VCT; GE Healthcare, Milwaukee, WI, USA) with a 5-mm slice width were used. Brain CT images were reviewed by two independent neurologists. Investigators who were blinded to clinical information opened these $\mathrm{CT}$ scans for each patient using commercial image-viewing software (Centricity RA1000 PACS Viewer; GE Healthcare, Milwaukee, WI, USA). ONSD and eyeball transverse diameter (ETD) were measured using the same initial CT and subsequent scans. The ONSD was measured at a distance of $3 \mathrm{~mm}$ behind the eyeball, immediately below the 
sclera in a perpendicular vector with reference to the linear axis of the nerve (Fig. 1A) ${ }^{6,9,22)}$. Images were changed to the "chest/ abdomen" window (window width $300 \&$ window level 10) and magnified four-fold on the particular image slice that demonstrated the largest diameter of the optic nerve sheath ${ }^{22)}$. The ONSD was measured from one side of the optic nerve sheath to the other as a section through the center of the optic nerve ${ }^{9)}$. The transverse diameter of the eyeball was chosen because the ONSD was usually measured in the transverse plane ${ }^{24)}$. ETD was defined as the maximal transverse diameter of the eyeball measured from one side of the retina to the other (in-to-in, Fig. 1B) ${ }^{1,24)}$. ONSD erage and the $\mathrm{ETD}_{\text {average }}$ measured for the patient's left and right eyes were averaged to yield mean values ${ }^{9}$. $\mathrm{ONSD}_{\text {index }}$ was defined as median ETD $(23.0 \mathrm{~mm})$ multiplied by the average value of bilateral ONSDs divided by the average value of bilateral ETDs $\left(23.0 \times \mathrm{ONSD}_{\text {average }} / \mathrm{ETD}_{\text {average }}\right)$.

\section{Resuscitation procedure}

CPR was led by the hospital's CPR team. All data related to the CPR scene were recorded by a bedside nurse according to the Utstein-style guidelines ${ }^{3)}$. The on-call ECMO team leader was called when CPR was performed for longer than 10 minutes or in the event of unstable vital signs or recurrent cardiac arrest. The ECMO team leader along with the CPR leader assessed the patient and decided whether to institute ECPR. ECPR was performed when a witnessed arrest was confirmed, the arrest per- sisted despite at least 10 minutes of conventional CPR, and the underlying cause of the arrest was considered reversible ${ }^{15}$. Cases in which ECPR was deferred included a short life expectancy ( $<6$ months), terminal malignancy, an unwitnessed collapse, limited physical activity, an unprotected airway, or CPR undertaken for longer than 60 minutes at the time of initial contact. Age alone did not constitute a contraindication of $\mathrm{ECPR}^{15)}$. The ECMO team at our institution consisted of cardiologists, cardiovascular surgeons, intensivists, specialized nurses, and perfusionists. Either a Capiox Emergency Bypass System (Terumo, Tokyo, Japan) or a Prolonged Life Support System (Maquet Cardiopulmonary, Hirrlingen, Germany) was used in these cases. A crystalloid solution such as normal saline or balanced solution was used for priming. No patient in this study underwent blood-primed ECMO. A percutaneous vascular approach was tried initially in all cases using the Seldinger technique. If percutaneous cannulation failed, a surgical cutdown exposure was performed ${ }^{15}$. Femoral vessels were the most common sites of vascular access and 14 to 17 French arterial cannulas and 20 to 24 French venous cannulas were placed $^{13}$. Cardiac compression was stopped once ECMO initiation was deemed successful. Anticoagulation was accomplished with a bolus injection of unfractionated heparin followed by a continuous intravenous heparin infusion to maintain an activated clotting time between 150 and 180 seconds. The initial number of revolutions per minute of the ECMO device was adjusted to achieve an ideal cardiac index greater than
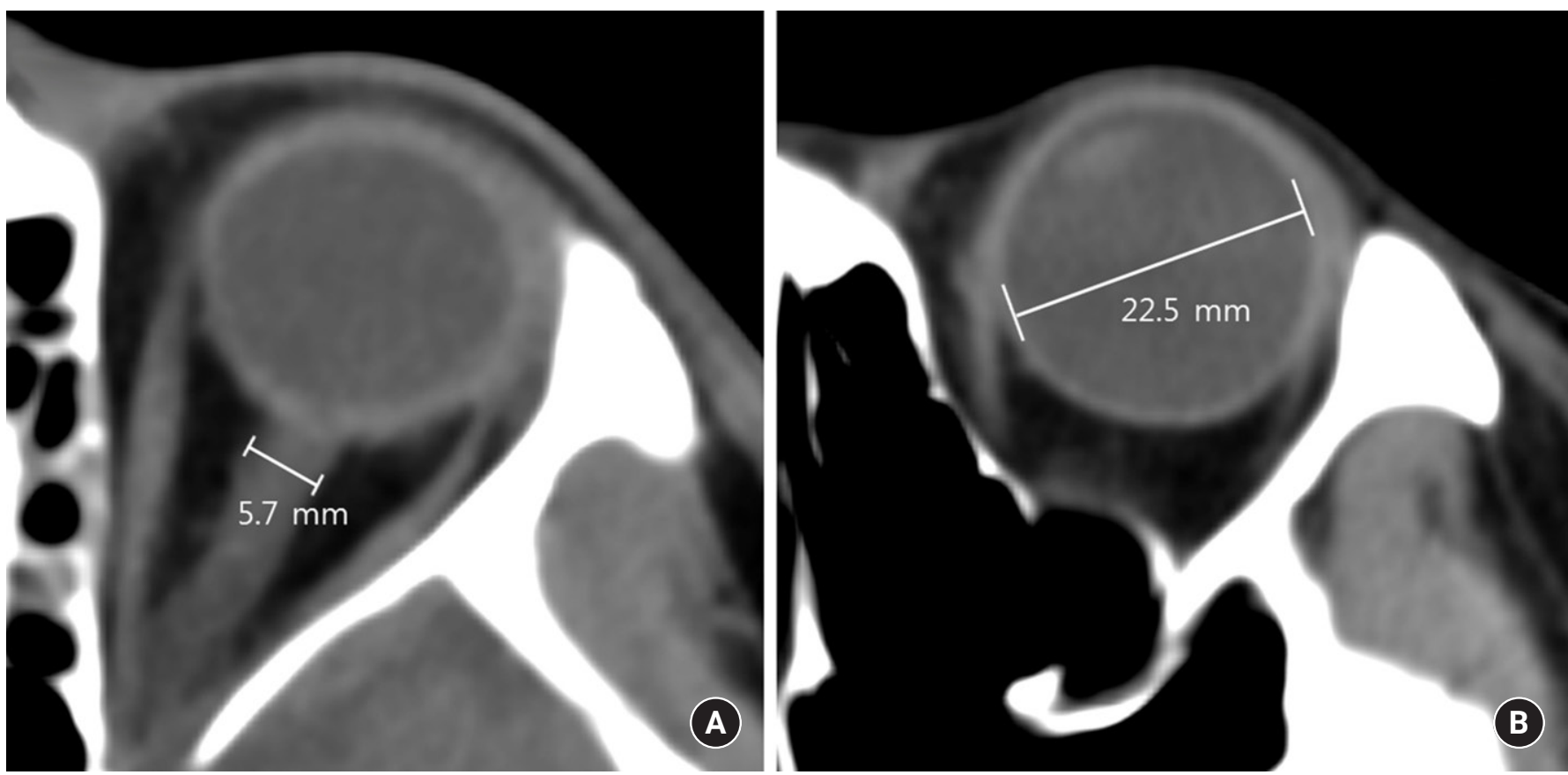

Fig. 1. A: Measurement of optic nerve sheath diameter. B: Eyeball transverse diameter on brain computed tomography scan. 
$2.2 \mathrm{~L} / \mathrm{min} / \mathrm{m}^{2}$ of body surface area, a central mixed venous oxygen saturation above $70 \%$, and a mean arterial pressure above 65 $\mathrm{mmHg}{ }^{13)}$. Blood pressure was monitored continuously through an arterial catheter and an artery in the right arm was used for arterial blood gas analysis to estimate cerebral oxygenation. After ECMO was established, necessary steps were taken to treat the underlying cause of cardiac arrest, including percutaneous coronary intervention, coronary artery bypass grafting, heart transplantation, and non-cardiopulmonary surgery ${ }^{13)}$.

\section{Statistical analyses}

All data are presented as medians and interquartile ranges (IQRs) for continuous variables and numbers (percentages) for categorical variables. Data were compared using Mann-Whitney $\mathrm{U}$ test for continuous variables and Chi-square test or Fisher's exact test for categorical variables. Predictive performances of ONSDs and their modification were assessed using area under the curve (AUC) of the receiver operating characteristic (ROC) curves for sensitivity vs. 1-specificity. AUCs were compared using the nonparametric approach published by DeLong et al. ${ }^{5)}$ for two correlated AUCs. Optimal cut-off values of ONSDs for predicting poor neurological outcome were obtained by ROC curve and Youden index ${ }^{14,20)}$. All tests were two-sided and p-values $<0.05$ were considered statistically significant. Data were ana- lyzed using IBM SPSS statistics version 20 (IBM, Armonk, NY, USA).

\section{RESULTS}

\section{Baseline characteristics and clinical outcomes}

A total of 52 patients with cardiac arrest who were rescued by veno-arterial ECMO were analyzed. Their median age was 53.0 years (IQR: 40.5-64.5 years). Of 52 patients, 39 (75.0\%) were males. A cardiac cause of arrest was verified in 46 (88.5\%) patients. Thirty-nine (75.0\%) patients experienced cardiac arrest in the hospital while 13 (25.0\%) patients had cardiac arrest in an out-of-hospital setting. The median time from arrest to ECMO pump-on was 43.0 minutes (IQR: 27.0-53.0 minutes). TTM was performed in 25 (48.1\%) patients using surface cooling devices. Baseline characteristics of ECPR patients are presented in Table 1. Characteristics of cardiac arrest are shown in Table 2. There were no significant differences in baseline or arrest-related characteristics between the two neurological outcome groups except hemoglobin level before ECMO. Successful ECMO weaning was achieved in 29 (55.8\%) patients. Survival to discharge was identified in 19 (36.5\%) patients. Of these 19 survivors, 13 (25.0\%) had good neurological outcomes (CPC score of 1 or 2 ).

Table 1. Baseline characteristics of patients

\begin{tabular}{|c|c|c|c|}
\hline & Good neurological outcome $(n=13)$ & Poor neurological outcome $(n=39)$ & p-value \\
\hline Age $(y r)$ - median $(\mathrm{IQR})$ & $50.0(34.0-54.0)$ & $54.0(46.0-64.5)$ & 0.224 \\
\hline Gender, male — no. of patients (\%) & $11.0(84.6)$ & $28.0(71.8)$ & 0.579 \\
\hline Body mass index $(\mathrm{kg} / \mathrm{m} 2)$ & $23.1(21.3-25.7)$ & $25.4(23.4-28.4)$ & 0.053 \\
\hline \multicolumn{4}{|l|}{ Medical history — no. of patients (\%) } \\
\hline Hypertension & $3.0(23.1)$ & $15.0(38.5)$ & 0.501 \\
\hline Current smoker & $5.0(38.5)$ & $12.0(30.8)$ & 0.864 \\
\hline Diabetes mellitus & $2.0(15.4)$ & $8.0(20.5)$ & 0.999 \\
\hline Malignancy & $1.0(7.7)$ & $9.0(23.1)$ & 0.416 \\
\hline Previous myocardial infarction & $0.0(0.0)$ & $4.0(10.3)$ & 0.548 \\
\hline \multicolumn{4}{|l|}{$\begin{array}{l}\text { Target temperature management }- \text { no. of } \\
\text { patients }(\%)\end{array}$} \\
\hline Arctic Sun & $6.0(46.2)$ & $13.0(33.3)$ & 0.531 \\
\hline Cooling pad & $2.0(15.4)$ & $4.0(10.3)$ & \\
\hline Initial Glasgow Coma Scale - median (IQR) & $3.0(3.0-6.0)$ & $3.0(3.0-4.0)$ & 0.834 \\
\hline \multicolumn{4}{|l|}{ Laboratory data on admission } \\
\hline Initial lactate $(\mathrm{mmol} / \mathrm{L})$ & $12.3(8.8-14.5)$ & $8.7(6.2-12.8)$ & 0.266 \\
\hline Hemoglobin before ECMO $(\mathrm{g} / \mathrm{dL})$ & $15.5(14.4-15.7)$ & $12.8(10.9-14.1)$ & 0.016 \\
\hline Hemoglobin after ECMO (g/dL) & $11.9(10.5-12.8)$ & $10.5(8.8-12.9)$ & 0.631 \\
\hline Total bilirubin $(\mathrm{mg} / \mathrm{dL})$ & $0.8(0.4-0.8)$ & $0.8(0.6-1.1)$ & 0.270 \\
\hline Blood urea nitrogen $(\mathrm{mg} / \mathrm{dL})$ & $15.6(13.6-16.9)$ & $15.5(12.4-23.3)$ & 0.695 \\
\hline Creatinine $(\mathrm{mg} / \mathrm{dL})$ & $1.1(0.9-1.4)$ & $1.1(1.0-1.5)$ & 0.769 \\
\hline Bicarbonate (IU/L) & $11.7(10.3-17.9)$ & $17.8(13.0-20.7)$ & 0.089 \\
\hline
\end{tabular}

IQR: interquartile range; ECMO: extracorporeal membrane oxygenation. 


\section{Optic nerve sheath diameters and their modifications}

In this study, ONSDs and ONSDindex in the poor neurological outcome group were significantly greater than those in the good neurological outcome group (Table 3). However, ETD was not significantly different between the two groups $(\mathrm{p}=0.770)$. In ROC curve analysis for predicting poor neurological outcome (Fig. 2), the C-statistic of $\mathrm{ONSD}_{\text {average }}$ was 0.856 (95\% CI: 0.731 to 0.938 ). A cut-off $>5.79 \mathrm{~mm}$ had a sensitivity of $71.8 \%$ ( $95 \%$ CI: $55.1 \%$ to $85.0 \%$ ) and a specificity of $92.3 \%$ (95\% CI: $64.0 \%$ to $99.8 \%$ ). The C-statistic of ONSD max $_{\text {ax }}$ wa.778 (95\% CI: 0.641 to 0.882 ). A cut-off $>6.24 \mathrm{~mm}$ had a sensitivity of $51.3 \%$ ( $95 \%$ CI: $34.8 \%$ to $67.6 \%$ ) and a specificity of $100 \%$ (95\% CI: $75.3 \%$ to $100 \%)$. The C-statistic of $\mathrm{ONSD}_{\text {index }}$ was 0.860 (95\% CI: 0.736 to 0.941 ). A cut-off $>5.91$ had a sensitivity of $71.8 \%$ (95\% CI: $55.1 \%$ to $85.0 \%$ ) and a specificity of $100 \%$ (95\% CI: $75.3 \%$ to $100 \%)$. However, ONSDs and their modification showed sim- ilar performances for predicting poor neurological outcome. The predictive performance of $\mathrm{ONSD}_{\text {index }}$ for poor neurological outcome was not significantly different from that of $\mathrm{ONSD}_{\text {average }}$ or $\mathrm{ONSD}_{\text {max }}(\mathrm{p}=0.913$ and $\mathrm{p}=0.138$, respectively $)$.

\section{DISCUSSION}

In the present study, we evaluated whether ONSD with their modification could be used to predict neurological outcomes in patients after ECPR. Major findings of this study were as follows: 1) ONSDs and $\mathrm{ONSD}_{\text {index }}$ in the poor neurological outcome group were significantly greater than those in the good neurological outcome group. These markers might predict neurological outcomes of patients after ECPR; 2) ETD was not significantly different between the two groups with different neurological outcomes; 3) Analysis of ROC curves for prediction of poor

Table 2. Characteristics of patients with cardiac arrest and procedures

\begin{tabular}{|c|c|c|c|}
\hline & Good neurological outcome $(n=13)$ & Poor neurological outcome $(n=39)$ & p-value \\
\hline Type of cardiac arrest — no. of patients (\%) & & & 0.999 \\
\hline Out of hospital cardiac arrest & $3.0(23.1)$ & $10.0(25.6)$ & \\
\hline Bystander witnessed cardiac arrest — no. of patients (\%) & $13.0(100.0)$ & $39.0(100.0)$ & \\
\hline Bystander performed CPR — no. of patients (\%) & $11.0(84.6)$ & $38.0(97.4)$ & 0.303 \\
\hline First monitored rhythm — no. of patients (\%) & & & 0.177 \\
\hline Pulseless electrical activity & $4.0(36.4)$ & $16.0(43.2)$ & \\
\hline Shockable rhythm (VT or VF) & $6.0(54.5)$ & $10.0(27.0)$ & \\
\hline Defibrillation — no. of patients (\%) & $7.0(58.3)$ & $17.0(45.9)$ & 0.679 \\
\hline Arrest to ECMO pump-on time — median (IQR) & $43.0(17.0-51.0)$ & $42.5(30.0-53.0)$ & 0.502 \\
\hline Location of ECMO insertion — no. of patients (\%) & & & 0.718 \\
\hline Other & $0.0(0.0)$ & $3.0(7.7)$ & \\
\hline Cardiac cause of arrest — no. of patients (\%) & & & 0.516 \\
\hline Ischemic & $3.0(25.0)$ & $14.0(41.2)$ & \\
\hline Non-ischemic & $9.0(75.0)$ & $20.0(58.8)$ & \\
\hline
\end{tabular}

CPR: cardiopulmonary resuscitation; VT: ventricular tachycardia; VF: ventricular fibrillation; ECMO: extracorporeal membrane oxygenation; IQR: interquartile range.

Table 3. Optic nerve sheath diameters and their modifications according to neurological outcomes

\begin{tabular}{lccc}
\hline & Good neurological outcome $(\mathbf{n}=\mathbf{1 3})$ & Poor neurological outcome $(\mathbf{n}=39)$ & p-value \\
ONSDaverage & $5.43(5.20-5.70)$ & $6.14(5.80-6.65)$ & $<0.001$ \\
ONSDmax & $5.68(5.39-6.10)$ & $6.41(5.85-6.83)$ & 0.003 \\
ETDaverage & $22.50(21.80-24.00)$ & $23.10(22.1-23.40)$ & 0.770 \\
ONSDindex & $5.62(5.34-5.87)$ & $6.29(5.86-6.54)$ & $<0.001$
\end{tabular}

ONSD: optic nerve sheath diameter; ETD: eyeball transverse diameter. 


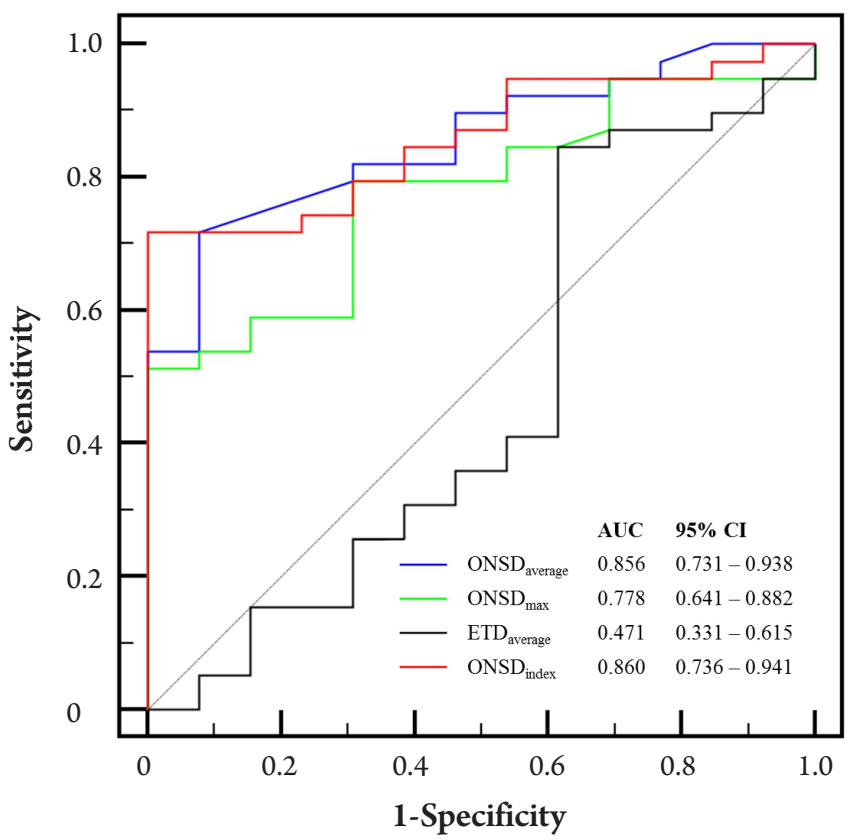

Fig. 2. Receiver operating characteristic curves for prediction of poor outcomes using optic nerve sheath diameters and their modifications. $\mathrm{ONSD}_{\text {index }}$ was defined as median ETD $(23.0 \mathrm{~mm})$ multiplied by the average value of bilateral ONSDs divided by the average value of bilateral ETDs $\left(23.0 \times \mathrm{ONSD}_{\text {average }} / \mathrm{ETD}_{\text {average }}\right)$. AUC: area under the curve; CI: confidence interval; ONSD: optic nerve sheath diameter; ETD: eyeball transverse diameter.

neurological outcome showed that ONSDs and $\mathrm{ONSD}_{\text {index }}$ had considerable predictive values and similar predictive performances for poor neurological outcome.

Primary cerebral injury is caused by immediate cessation of cerebral blood flow following cardiac arrest that results in neuron ischemia and brain cell death ${ }^{21)}$. In addition, secondary cerebral injury can occur and cause additive cerebral injury characterized by an imbalance in post-resuscitation cerebral oxygen delivery and use, ultimately culminating in neuronal death ${ }^{21}$. This injury begins immediately after ROSC, including reperfusion injury, microcirculatory dysfunction, impaired cerebral autoregulation, refractory intracranial hypertension, hypoxemia, hyperoxia, hyperthermia, fluctuations in arterial carbon dioxide, and concomitant anemia ${ }^{21)}$. Several pathophysiologic mechanisms involved in the increased risk of intracranial hypertension after cardiac arrest has been suggested. Blood brain barrier alterations have been demonstrated after global ischemia ${ }^{6}$. Impaired autoregulation and post-ischemic hyperperfusion associated with global ischemia may also increase the risk of ICP elevation ${ }^{6}$. Eventually, primary and secondary injuries are associated with intracranial hypertension.

ONSD has been proposed as an alternative parameter for de- tection of increased $\mathrm{ICP}^{6}$. The optic nerve is surrounded by cerebrospinal fluid because it is a part of the central nervous system. Therefore, increased ICP is transmitted through the subarachnoid space surrounding the optic nerve within the nerve sheath, especially the retrobulbar segment, unless circulation of cerebrospinal fluid is not blocked ${ }^{6}$. ONSD measured during the initial brain CT may be correlated with neurologic outcomes after traumatic brain injury ${ }^{22,23)}$. Simultaneous measurement of ONSD with initial CT and ICP are correlated and ONSD is indicative of intracranial hypertension in patients with severe traumatic brain injury $^{22,23)}$.

Although ONSD is considered an indirect marker for ICP, its optimal cut-off for an abnormal ONSD indicating elevated ICP and its associated factors have been unclear ${ }^{8}$. In addition, there are limited studies reporting the role of ONSD modifications or indices in detecting intracranial hypertension compared with absolute $\mathrm{ONSD}^{1,24)}$. ONSD correlates strongly with ETD in healthy people. ONSD/ETD ratio may provide highly reliable data than absolute ONSD as a marker of $\mathrm{ICP}^{8)}$. However, in this study, there were no significant differences between ONSD/ETD ratio and absolute ONSD for predicting poor neurological outcomes. Therefore, future studies with larger cohorts are needed to confirm these findings.

This study has several limitations. First, the CPC scale was retrospectively determined based on medical records. Second, the nonrandomized nature of the registry data might have resulted in selection bias. Although brain CT scans were performed within 48 hours following ECPR, a major limitation of this study might be that CT scans were performed at different time settings. Third, ONSD measurement requires high image resolution and specific CT settings such as optimal slice thickness and slice angle with the skull base. The heterogeneity of CT scan protocols might have led to the relatively weak correlation between ONSD and ICP in this study ${ }^{10}$. Fourth, a limited number of patients received targeted temperature management. Methods used were decided by each intensivist. They varied according to conditions and situations. Finally, our study had limited statistical power due to its small sample size. Although it still provides valuable insight, prospective large-scale studies are needed to evaluate the usefulness of ONSD based on brain CT for predicting neurological outcomes of patients after ECPR to obtain evidence-based conclusions.

\section{CONCLUSION}

In this study, ONSDs measured with CT may be used to predict neurological outcomes of ECPR survivors. 


\section{CONFLICT OF INTEREST}

No potential conflict of interest relevant to this article was reported.

\section{REFERENCES}

1. Bekerman I, Sigal T, Kimiagar I, Ben Ely A, Vaiman M. The quantitative evaluation of intracranial pressure by optic nerve sheath diameter/eye diameter CT measurement. Am J Emerg Med 2016;34:2336-2342.

2. Chen YS, Lin JW, Yu HY, Ko WJ, Jerng JS, Chang WT, et al. Cardiopulmonary resuscitation with assisted extracorporeal life-support versus conventional cardiopulmonary resuscitation in adults with in-hospital cardiac arrest: an observational study and propensity analysis. The Lancet 2008;372:554 561.

3. Cummins RO, Chamberlain D, Hazinski MF, Nadkarni V, Kloeck W, Kramer E, et al. Recommended guidelines for reviewing, reporting, and conducting research on in-hospital resuscitation: the in-hospital 'Utstein style'. American Heart Association. Circulation 1997;95:2213-2239.

4. Cummins RO, Chamberlain DA, Abramson NS, Allen M, Baskett PJ, Becker L, et al. Recommended guidelines for uniform reporting of data from out-of-hospital cardiac arrest: the Utstein Style. A statement for health professionals from a task force of the American Heart Association, the European Resuscitation Council, the Heart and Stroke Foundation of Canada, and the Australian Resuscitation Council. Circulation 1991;84:960-975.

5. DeLong ER, DeLong DM, Clarke-Pearson DL. Comparing the areas under two or more correlated receiver operating characteristic curves: a nonparametric approach. Biometrics 1988;44:837-845.

6. Kim YH, Lee JH, Hong CK, Cho KW, Yeo JH, Kang MJ, et al. Feasibility of optic nerve sheath diameter measured on initial brain computed tomography as an early neurologic outcome predictor after cardiac arrest. Acad Emerg Med 2014;21:1121-1128.

7. Kang MJ, Lee TR, Shin TG, Sim MS, Jo IJ, Song KJ, et al. Survival and neurologic outcomes of out-of-hospital cardiac arrest patients who were transferred after return of spontaneous circulation for integrated post-cardiac arrest syndrome care: the another feasibility of the cardiac arrest center. J Korean Med Sci 2014;29:1301-1307.

8. Kim DH, Jun JS, Kim R. Ultrasonographic measurement of the optic nerve sheath diameter and its association with eye- ball transverse diameter in 585 healthy volunteers. Sci Rep 2017;7:15906.

9. Legrand A, Jeanjean P, Delanghe F, Peltier J, Lecat B, Dupont $H$. Estimation of optic nerve sheath diameter on an initial brain computed tomography scan can contribute prognostic information in traumatic brain injury patients. Crit Care 2013;17:R61.

10. Monnin P, Sfameni N, Gianoli A, Ding S. Optimal slice thickness for object detection with longitudinal partial volume effects in computed tomography. J Appl Clin Med Phys 2017;18:251-259.

11. Morimura N, Sakamoto T, Nagao K, Asai Y, Yokota H, Tahara Y, et al. Extracorporeal cardiopulmonary resuscitation for out-of-hospital cardiac arrest: A review of the Japanese literature. Resuscitation 2011;82:10-14.

12. Nagao K, Kikushima K, Watanabe K, Tachibana E, Tominaga Y, Tada K, et al. Early Induction of Hypothermia During Cardiac Arrest Improves Neurological Outcomes in Patients With Out-of-Hospital Cardiac Arrest Who Undergo Emergency Cardiopulmonary Bypass and Percutaneous Coronary Intervention. Circulation Journal 2010;74:77-85.

13. Park SB, Yang JH, Park TK, Cho YH, Sung K, Chung CR, et al. Developing a risk prediction model for survival to discharge in cardiac arrest patients who undergo extracorporeal membrane oxygenation. Int J Cardiol 2014;177:1031-1035.

14. Ruopp MD, Perkins NJ, Whitcomb BW, Schisterman EF. Youden Index and optimal cut-point estimated from observations affected by a lower limit of detection. Biom J 2008;50:419-430.

15. Ryu JA, Cho YH, Sung K, Choi SH, Yang JH, Choi JH, et al. Predictors of neurological outcomes after successful extracorporeal cardiopulmonary resuscitation. BMC Anesthesiol 2015; 15:26.

16. Ryu JA, Chung CR, Cho YH, Sung K, Suh GY, Park TK, et al. The association of findings on brain computed tomography with neurologic outcomes following extracorporeal cardiopulmonary resuscitation. Crit Care 2017;21:15.

17. Ryu JA, Park TK, Chung CR, Cho YH, Sung K, Suh GY, et al. Association between Body Temperature Patterns and Neurological Outcomes after Extracorporeal Cardiopulmonary Resuscitation. PLoS One 2017;12:e0170711.

18. Sakamoto T, Morimura N, Nagao K, Asai Y, Yokota H, Nara $S$, et al. Extracorporeal cardiopulmonary resuscitation versus conventional cardiopulmonary resuscitation in adults with out-of-hospital cardiac arrest: a prospective observational study. Resuscitation 2014;85:762-768.

19. Sandroni C, Cariou A, Cavallaro F, Cronberg T, Friberg H, 
Hoedemaekers C, et al. Prognostication in comatose survivors of cardiac arrest: an advisory statement from the European Resuscitation Council and the European Society of Intensive Care Medicine. Intensive Care Med 2014;40:18161831.

20. Schisterman EF, Perkins NJ, Liu A, Bondell H. Optimal cutpoint and its corresponding Youden Index to discriminate individuals using pooled blood samples. Epidemiology 2005;16:73-81.

21. Sekhon MS, Ainslie PN, Griesdale DE. Clinical pathophysiology of hypoxic ischemic brain injury after cardiac arrest: a “two-hit” model. Crit Care 2017;21:90.

22. Sekhon MS, Griesdale DE, Robba C, McGlashan N, Needham E, Walland $\mathrm{K}$, et al. Optic nerve sheath diameter on computed tomography is correlated with simultaneously measured intracranial pressure in patients with severe traumatic brain injury. Intensive Care Med 2014;40:1267-1274.

23. Sekhon MS, McBeth P, Zou J, Qiao L, Kolmodin L, Henderson WR, et al. Association between optic nerve sheath diameter and mortality in patients with severe traumatic brain injury. Neurocrit Care 2014;21:245-252.

24. Vaiman M, Gottlieb P, Bekerman I. Quantitative relations between the eyeball, the optic nerve, and the optic canal important for intracranial pressure monitoring. Head Face Med 2014; 10:32.

25. Young GB. Clinical practice. Neurologic prognosis after cardiac arrest. N Engl J Med 2009;361:605-611. 